\title{
Postpartum Catastrophic Anti-Phospholipid Syndrome
}

\author{
*EM Manoj ${ }^{1}$, WDD Priyankara ${ }^{1}$, DR Liyanagunawardana ${ }^{2}$, S Jayasinghe ${ }^{3}$ \\ Senior Registrar in Critical care medicine ${ }^{1}$, Senior Registrar in Anaesthesiology ${ }^{2}$, National Hospital of \\ Sri Lanka, Consultant Anaesthetist ${ }^{3}$, De Soyza Hospital for Women, Sri Lanka.
}

*Corresponding author: manojediri@yahoo.com

\begin{abstract}
Multi-organ dysfunction during peripartum period in the background of anti-phospholipid syndrome (APS) poses a tremendous diagnostic dilemma leading to extremely high morbidity and mortality. We present a diagnosed APS case of 29 year old multigravida, who was admitted to intensive care unit with post-partum pulmonary edema, severe thrombocytopenia, paralytic ileus, hepatic and splenic infarcts, status epilepticus and lower limb venous thrombosis despite being on prophylactic anticoagulation. She recovered after treatment with escalating doses of prednisolone, antiepileptics, mechanical ventilator support with muscle paralysis and therapeutic plasma exchange (TPE).This case highlights the utmost importance of awareness, high degree of suspicion and prompt adoption of appropriate supportive treatment to achieve a favourable outcome in catastrophic anti phospholipid syndrome (CAPS).
\end{abstract}

Key words: catastrophic anti phospholipid syndrome, thrombotic thrombocytopenic purpura, therapeutic plasma exchange, hepatic infarcts, HELLP syndrome

\section{Introduction}

Anti-phospholipid syndrome poses a tremendous threat during pregnancy, not only due to its thrombotic tendency but with associated complications such as pre-eclamptic toxaemia, HELLP syndrome, etc. APS is an autoimmune disease with a misleading name, because the pathologic autoantibodies are directed against the plasma protein - glycoprotein and not against phospholipids. ${ }^{1}$ Exceptionally, the multi-organ dysfunction (MODS) secondary to CAPS in this context carries an extremely high morbidity and mortality. Herein, we present a case of postpartum CAPS which was successfully managed, highlighting the importance of anticipation and supportive therapy.

\section{Case report}

A 29 year old woman (gravida 6 para 0) presented with preterm premature rupture of membranes (PPROM) at 33 weeks of gestation. She was diagnosed as having APS during the antenatal period based on the clinical criteria of recurrent miscarriages as well as the positivity of anticardiolipin (IgG) antibodies. Subsequently, she had been on prophylactic dose of low molecular weight heparin (LMWH). Eventually, she underwent emergency caesarian section
(LSCS)due to poor progression of labour giving birth to a healthy baby.

Her post-partum period was complicated with sudden onset backache, respiratory distress with transient desaturation, progressive abdominal distension with evidence of paralytic ileus and worsening thrombocytopenia by the $3^{\text {rd }}$ day. Her contrast enhanced CT scan revealed multiple hepatic infarcts but evidence for obvious bowel pathology was absent. (Figure 1) Workup for pulmonary embolism was negative but treatment with therapeutic dose of LMWH anticoagulation, prednisolone and intravenous meropenem was started. Next day, she had an episode of status epilepticus followed by severe pulmonary edema. As a result she underwent endotracheal intubation, ventilatory support, escalating doses of antiepileptics, pharmacological paralysis and diuresis with a favourable outcome. Considering the course of the illness, CAPS was suspected and she was further treated with daily cycles of TPE for 5 days. Repeat CT of abdomen showed extension of thrombosis to the other segments of liver and to the spleen as well. She was gradually weaned off and extubated after 3 days of ventilator support and she developed deep venous thrombosis in the left leg on eighth postpartum day. She was discharged on $16^{\text {tht }}$ postpartum day 
with a plan for lifelong anticoagulation and tapering of prednisolone.

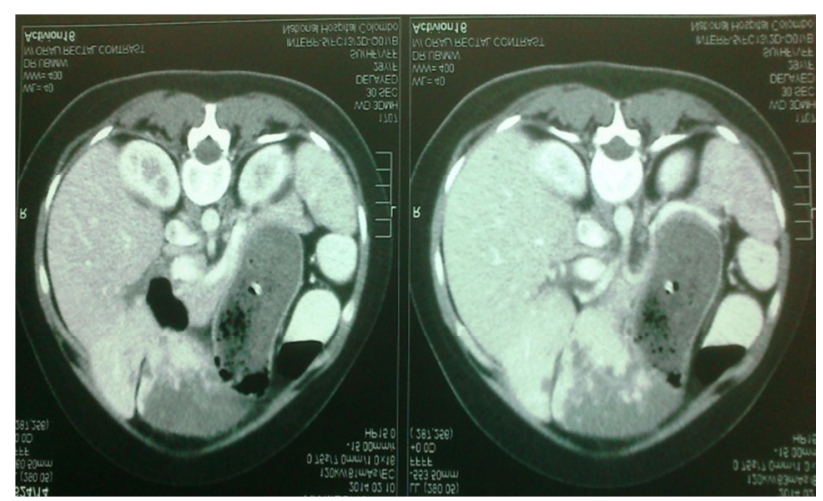

Figure 1; Abdominal CT showing hepatic infarctions involving segment II and III. Repeat $\mathrm{CT}$; New infarctions in segment IV of liver and a wedge shaped hypo dense area in spleen suggestive of new infarct

\section{Discussion}

Catastrophic anti phospholipid syndrome, described by Asherson in 1992, is a rare form of APS with a mortality rate of about $50 \%$. $^{2}$ CAPS during pregnancy or puerperium represents almost $6 \%$ of all cases described.Large vessel occlusions do not dominate the clinical picture in CAPS as opposed to classic APS. Instead, CAPS is characterized by thrombotic occlusion of the microvasculature with liberation of cytokines which lead to further disturbance of vascular endothelial cell function and severe MODS. ${ }^{3}$ The kidney is the organ most commonly affected, followed by the lung, central nervous system, heart and disseminated intravascular coagulation(DIC) occurs in approximately $13 \%$ of patients. "Trigger" factors have been demonstrated in $45 \%$ of patients, but in the majority, they remain unknown. Compelling evidence suggests that surgery, trauma, infections, and malignancy can result in a conformational change in the anti- $\bullet$ beta 2-glycoprotein I antibody complex. This can then interact with endothelial surface receptors $\bullet$ such as Annexin A2 and LRP8 and induce prc $\bullet$ inflammatory and micro thrombotic phenomena. ${ }^{1}$ Interestingly, our patient did not have a demonstrable renal involvement but had evidence of extensive micro thrombotic phenomena in the form of MODS as well as large venous thrombosis evidenced by the involvement of leg veins.

Women with APS seem to have increased risk of developing a HELLP syndrome. Pauzner et al., found liver infarcts to be almost always associated with APS in women with HELLP syndrome. ${ }^{4}$ For histopathological confirmation, thrombosis should be present without evidence of inflammation in the vessel wall. ${ }^{5}$ Even though, the liver enzymes were moderately elevated in our patient along with multiple hepatic infarcts, absence of other features did not favor HELLP syndrome as the primary diagnosis.

APS related thrombocytopenia which is defined as the platelet count less than $100 \times 10^{9} / \mathrm{L}$, confirmed twice 12 weeks apart, is an autoimmune phenomenon and its relation to thrombotic risk is poorly characterized. The other differential diagnoses in patients presenting with thrombocytopenia includes thrombotic thrombocytopenic purpura (TTP), heparin-induced thrombocytopenia (HIT) and DIC. ${ }^{6}$ Distinguishing these conditions can be challenging. Since our patient was under medication with LMWH, though the incidence is much less, HIT must be considered. However, she did not manifest significant bleeding manifestations or red-cell fragments in blood film making the probability of DIC or TTP low. On the other hand, distinguishing TTP from CAPS apparently was not that crucial for the acute management as plasma exchange is the mainstay of therapy in either condition.

There is a paucity of evidence based recommendations on management of CAPS. If CAPS is suspected, aggressive supportive treatment with a goal to achieve following should be started without delay. ${ }^{7}$

To treat any "trigger" factors (e.g., early antibiotics if infection is suspected).

To prevent and to treat existing blood clots.

To suppress excessive inflammation eg; corticosteroids, TPE

In conclusion, the catastrophic anti-phospholipid syndrome might be readily overlooked unless high degree of suspicion is present. MODS is difficult 
to be attributed to either the sepsis or the APLS in the acute stage highlighting the importance of aggressive supportive care. Early diagnosis of CAPS in critically ill patients who have a history of APS is extremely important for a favourable outcome.

\section{References}

1. de Groot PG, Urbanus RT, The significance of autoantibodies against 2-glycoprotein I: Blood, 2012; 20 (2): 266- 274.

http://dx.doi.org/10.1182/blood-2012-03-378646

PMid:22553312

2. Asherson RA. The catastrophic antiphospholipid syndrome: Journal of Rheumatology: 1992;19 (4): 508-512.

PMid:1593568

3. Praprotnik S, Ferluga D, Vizjak A. Microthrombotic/microangiopathic manifestations of the antiphospholipid syndrome. Clinical Reviews in Allergy and Immunology. 2009; 36 (23): 109-125.

http://dx.doi.org/10.1007/s12016-008-8104-z PMid:19067253

4. Pauzner R, Dulitzky M, Carp H. Hepatic infarctions during pregnancy are associated with the antiphospholipid syndrome and in addition with complete or incomplete HELLP syndrome. J ThrombHaemost .2003;1(8):1758-63.

http://dx.doi.org/10.1046/j.15387836.2003.00253.x

PMid:12911590

5. Miyakis S, Lockshin MD, Atsumi T. International consensus statement on an update of the classification criteria for definite antiphospholipid syndrome. J ThrombHaemost : 2006 ; 4 (2):295306.

http://dx.doi.org/10.1111/j.15387836.2006.01753.x PMid:16420554

6. Lim W. Antiphospholipid antibody syndrome. Hematology- Am SocHematolEduc Program 2009; 233-9.

http://dx.doi.org/10.1182/asheducation-2009.1.233 PMid:20008203

7. Erkan D. Therapeutic and prognostic considerations in catastrophic antiphospholipid syndrome Autoimmunity Reviews 2006; 6: 98103.

http://dx.doi.org/10.1016/j.autrev.2006.06.013

PMid:17138252 